\title{
The Averaging Principle for Stochastic Fractional Partial Differential Equations with Fractional Noises
}

\author{
JING Yuanyuan, LI Zhi and XU Liping* \\ School of Information and Mathematics, Yangtze University, Jingzhou 434023, China.
}

Received 1 June 2020; Accepted 15 July 2020

\begin{abstract}
The purpose of this paper is to establish an averaging principle for stochastic fractional partial differential equation of order $\alpha>1$ driven by a fractional noise. We prove the existence and uniqueness of the global mild solution for the considered equation by the fixed point principle. The solutions for SPDEs with fractional noises can be approximated by the solution for the averaged stochastic systems in the sense of $p$-moment under some suitable assumptions.
\end{abstract}

AMS Subject Classifications: 26A33; 60G15; 60H15

Chinese Library Classifications: O211

Key Words: Averaging principle; Stochastic fractional partial differential equation; fractional noises.

\section{Introduction}

Fractional calculus has attracted many physicists, mathematicians and engineers due to the contributions which have been made to both theory and applications of fractional (partial) differential equations(see, e.g., [1] and references therein). Mueller [2] and $\mathrm{Wu}$ [3] proved the existence of a solution of the stochastic fractional heat equation. Then, Bonaccorsi and Tubaro [4] applied Mittag-Leffler's function to explore stochastic evolution equations with fractional time derivatives. After that, Cui and Yan [5] studied the existence of mild solutions for a class of fractional neutral stochastic integro-differential equations with infinite delay in Hilbert spaces; In [6], Liu and Yan have established the existence and uniqueness of solutions to a jump-type stochastic fractional partial differential equation with fractional noises by fixed point theorem.

\footnotetext{
*Corresponding author. Email addresses: xlp211@126.com (L. P. Xu), lizhi_csu@126.com (Z. Li), jingyy120920@163.com (Y. Y. Jing) 
The averaging principle plays a crucial role to obtain the approximation solutions for differential equations dating from mechanics, molecular dynamics, mathematics, material science, and other areas of sciences and engineering. Some rigorous results on the approximation theorem to the solutions of stochastic differential equations can be dated back to Khasminskii [7,8]. Based on this work, recently, Xu et al. [9] have established the averaging principle for the solutions of stochastic partial differential equations driven by Lévy noise under Lipschitz and linear growth conditions. Peculiarly, they have proved that the solutions to the simplified systems converge to that of the corresponding original systems both in the sense of mean square and probability. Similar results were proposed to the multivalued stochastic differential equations by [10]. Not only that, Pei et al. [11] established the averaging principles for a class of stochastic partial differential equations with slow component driven by fractional Brownian motion and a fast one driven by a fast-varying diffusion. In case with Poisson random measure was studied in Pei et al. [12] and $\alpha$-noise in Bao et al. [13]. An averaging principle for the heat equation driven by a general stochastic measure was studied by Radchenko [14].

On the other hand, there has been some recent interest in studying stochastic partial differential equations driven by a fractional noise. For example, Duncan et al. [15] considered linear stochastic evolution equations in a Hilbert space driven by an additive cylindrical fractional Brownian motion with $H \in\left(\frac{1}{2}, 1\right)$ and Tindel et al. [16] provided necessary and sufficient conditions for the existence and uniqueness of an evolution solution. Many interesting works on stochastic partial differential equations driven by $\mathrm{fBm}$ have been done and we refer to the literatures [17-19].

Based on the above brief discussion and to the author's best knowledge, the averaging principle for stochastic fractional partial differential equations with fractional noises has not been considered. Therefore, in this paper, we will consider this issue by studying the following stochastic fractional partial differential equation with fractional noises:

$$
\left\{\begin{array}{l}
\frac{\partial u}{\partial t}=D_{\delta}^{\alpha} u+f(t, x, u)+\sigma(t, x) \dot{B}^{H}, \quad \text { in }[0, T] \times \mathbb{R}, \\
u(0, \cdot)=u_{0}(\cdot),
\end{array}\right.
$$

where $D_{\delta}^{\alpha}$ is the fractional differential operator with respect to the spatial variable, to be defined in the Appendix which was recently introduced by Debbi [20] and Debbi and Dozzi [21], $\dot{B}^{H}$ denotes the fractional noise on $[0, T] \times \mathbb{R}$ with Hurst index $H>\frac{1}{2}$ defined on a complete probability space $(\Omega, \mathcal{F}, P)$ (see Section 2 for precise definitions). In fact, we understand this equation in the Walsh [22] sense, and so we can rewrite Eq. (1.1) as follows:

$$
\begin{gathered}
u(t, x)=\int_{\mathbb{R}} G_{\alpha}(t, x-y) u_{0}(y) \mathrm{d} y+\int_{0}^{t} \int_{\mathbb{R}} G_{\alpha}(t-s, x-y) \sigma(s, y) B^{H}(\mathrm{~d} s, \mathrm{~d} y) \\
+\int_{0}^{t} \int_{\mathbb{R}} G_{\alpha}(t-s, x-y) f(s, y, u(s, y)) \mathrm{d} y \mathrm{~d} s,
\end{gathered}
$$


for all $t \in[0, T]$ and $x \in \mathbb{R}$, where $G_{\alpha}(\cdot, *)$ denotes the Green function associated to Eq. (1.1).

We can notice that the fixed principle and the Picard iteration scheme work in [23-25], which can be used to prove the existence and uniqueness of solutions to Eq. (1.1). The first subject of this paper is to establish the existence and uniqueness of the solution of Eq. (1.2) via the fixed point principle. And then, we will consider the averaging principle for Eq. (1.2) under some appropriate assumptions.

The rest of the paper is organized as follows. In Section 2, we give the definitions of the fractional noises. Section 3 is devoted to proving the existence and uniqueness of the mild solution to Eq. (1.2) in the $L^{p}(p \geq 2)$ sense under some appropriate conditions. An averaging principle for solutions to stochastic fractional partial differential equation with fractional noises in Section 4.

\section{Preliminaries}

In this section, we present the definitions of the fractional noises (see Sec.2 in [6]).

Define $\left(\Omega, \mathcal{F},\left(\mathcal{F}_{t}\right)_{t \geq 0}, P\right)$ for a complete probability space equipped with the filtration $\left(\mathcal{F}_{t}\right)_{t \geq 0}$ satisfying the usual conditions. Let $\mathcal{B}_{b}(\mathbb{R})$ be a class of bounded Borel sets in $\mathbb{R}$. And $B^{H}([0, t] \times A)_{(t, A) \in[0, T] \times \mathcal{B}_{b}(\mathbb{R})}$ is a centered Gaussian family of random variables with the covariance, for $H \in(0,1)$,

$$
\mathbb{E}\left(B^{H}([0, t] \times A) B^{H}([0, s] \times B)\right)=|A \cap B| R_{H}(t, s),
$$

with $s, t \in[0, T], A, B \in \mathcal{B}_{b}(\mathbb{R})$ and covariance kernel $R_{H}(t, s)=\frac{1}{2}\left(t^{2 H}+s^{2 H}-|t-s|^{2 H}\right)$. Here $|A|$ denotes the Lebesgue measure of the set $A \in \mathcal{B}_{b}(\mathbb{R})$. We denote by $\mathcal{E}$ the set of step functions on $[0, T] \times \mathbb{R}$. Let $\mathcal{H}$ be the Hilbert space defined as the closure of $\mathcal{E}$ with respect to the scalar product,

$$
\left\langle 1_{[0, t] \times A}, 1_{[0, s] \times B}\right\rangle_{\mathcal{H}}=|A \cap B| R_{H}(t, s),
$$

thus the mapping $1_{[0, t]} \times A \longmapsto B^{H}([0, t] \times A)$ is an isometry between $\mathcal{E}$ and the linear space span of $B^{H}([0, t] \times A), A \in \mathcal{B}_{b}(\mathbb{R}), t \in[0, T]$. Moreover, the mapping can be extended to an isometry from $\mathcal{H}$ to the Gaussian space associated with $B^{H}$. This isometry will be denoted by $\varphi \longmapsto B^{H}(\varphi)$ for $\varphi \in \mathcal{H}$. Therefore, we can regard $B^{H}(\varphi)$ as the stochastic integral with respect to $B^{H}$. In general, we use the notation,

$$
B^{H}(\varphi)=\int_{0}^{T} \int_{\mathbb{R}} \varphi(s, y) B^{H}(\mathrm{~d} s, \mathrm{~d} y), \quad \varphi \in \mathcal{H} .
$$

On the other hand, it is well known that the covariance kernel $R_{H}(t, s)$ satisfies

$$
R_{H}(t, s)=\int_{0}^{t \wedge s} K_{H}(t, r) K_{H}(s, r) \mathrm{d} r
$$


where $K_{H}(t, s)$ is the square kernel defined, for $0<s<t$, by

$$
K_{H}(t, s)=c_{H} s^{\frac{1}{2}-H} \int_{s}^{t}(u-s)^{H-\frac{3}{2}} u^{H-\frac{1}{2}} \mathrm{~d} u,
$$

where $c_{H}^{2}=\frac{H(2 H-1)}{\beta\left(2-2 H, H-\frac{1}{2}\right)},\left(\beta(\cdot, \cdot)\right.$ denotes the Beta function). Particularly, for $H>\frac{1}{2}$,

$$
R_{H}(t, s)=H(2 H-1) \int_{0}^{t} \int_{0}^{s}|u-v|^{2 H-2} \mathrm{~d} u \mathrm{~d} v .
$$

Definition 2.1. Let $\mathcal{K}_{H}^{*}: \mathcal{E} \longmapsto L^{2}([0, T] \times \mathbb{R})$ be a linear operator which satisfies

$$
\left(\mathcal{K}_{H}^{*} \psi\right)(s, x)=K_{H}(T, s) \psi(s, x)+\int_{s}^{T}(\psi(t, x)-\psi(s, x)) \frac{\partial K_{H}}{\partial t}(t, s) \mathrm{d} t .
$$

Then the operator $\mathcal{K}_{H}^{*} \psi$ gives an isometry from $\mathcal{H}$ to $L^{2}([0, T] \times \mathbb{R})$. As a result,

$$
W(t, A)=B^{H}\left(\left(\mathcal{K}_{H}^{*}\right)^{-1} \cdot 1_{[0, t] \times A}\right), \quad(t, A) \in[0, T] \times \mathcal{B}_{b}(\mathbb{R}),
$$

defines a space-time white noise. Moreover, one can view $B^{H}$ as

$$
B^{H}([0, t] \times A)=\int_{0}^{t} \int_{A} K_{H}(t, s) W(\mathrm{~d} s, \mathrm{~d} y) .
$$

\section{Existence and uniqueness}

In this section, we discuss the existence and uniqueness of the global mild solution to Eq. (1.1).

Recall Eq. (1.2) and Definition 2.1, the fractional integral term in Eq. (1.2) can be expressed as

$$
\int_{0}^{t} \int_{\mathbb{R}} G_{\alpha}(t-s, x-y) \sigma(s, y) B^{H}(\mathrm{~d} s, \mathrm{~d} y)=\int_{0}^{t} \int_{\mathbb{R}}\left(\mathcal{K}_{H}^{*} G_{\alpha} \sigma\right)(t-\cdot, x-\cdot) W(\mathrm{~d} s, \mathrm{~d} y),
$$

with the space-time white noise $W(t, x)$ for all $(t, x) \in[0, T] \times \mathbb{R}$ mentioned in Section 2 .

Theorem 3.1. Let $\sigma \in L^{\frac{2}{2 H-1}}([0, T] \times \mathbb{R})$ with $H>\frac{1}{2}$, suppose that the following conditions are satisfied

(1) $f$ is uniformly Lipschitzian, i.e. there exists a constant $L>0$ such that for $(t, x) \in[0, T] \times \mathbb{R}$ and $u, v \in \mathbb{R}$,

$$
|f(t, x, u)-f(t, x, v)| \leq L|u-v| .
$$

(2) fhas linear growth on $\mathbb{R}$, i.e. there exists a constant $C>0$ such that $|f(t, x, u)| \leq C(1+|u|)$, for $(t, x) \in[0, T] \times \mathbb{R}$ and $u \in \mathbb{R}$. 
Then, for all $\mathcal{F}_{0}$-measurable $u_{0}: \mathbb{R} \times \Omega \longrightarrow \mathbb{R}$ satisfying $\mathbb{E}\left[\left\|u_{0}(\cdot)\right\|_{p}^{p}\right]<\infty$, there exists a unique mild solution $u(t, x)_{(t, x) \in[0, T] \times \mathbb{R}}$ to Eq. (1.1) and for all $p \in\left(\frac{2(\alpha+1)}{\alpha-1},+\infty\right)$ with $\alpha>1$,

$$
\sup _{t \in[0, T]} \mathbb{E}\left[\|u(t, \cdot)\|_{p}^{p}\right]<\infty .
$$

In order to prove the theorem, we need the following two useful Lemmas:

Lemma 3.1. ([6]) Let $p \in[1, \infty), \rho \in[1, p]$ and $r \in[1, \infty)$ such that

$$
\frac{1}{r}=\frac{1}{p}-\frac{1}{\rho}+1 \in[0,1] \text {. }
$$

Let $G_{\alpha}=G_{\alpha}(t, x-y)$ be the Green kernel, $\pi=G_{\alpha}$, or $G_{\alpha}^{2}$ with $(t, x, y) \in[0, T] \times \mathbb{R} \times \mathbb{R}$. Define an operator J by

$$
J(v)(t, x):=\int_{0}^{t} \int_{\mathbb{R}} \pi(t-s, x-y) v(s, y) \mathrm{d} y \mathrm{~d} s,
$$

with $v \in L^{1}\left([0, T] ; L^{\rho}\right)$. Then $J: L^{1}\left([0, T] ; L^{\rho}\right) \longrightarrow L^{\infty}\left([0, T] ; L^{\rho}\right)$ is a bounded linear operator and satisfies the following.

(1) If $\pi=G_{\alpha}$, then there exists a constant $C>0$ such that for all $r \in[1,1+\alpha)$,

$$
\|J(v)(t, \cdot)\|_{p} \leq C \int_{0}^{t}(t-s)^{-\frac{1-r}{\alpha}}\|v(s, \cdot)\|_{\rho} \mathrm{d} s, \quad \forall t \in[0, T] .
$$

(2) If $\pi=G_{\alpha}^{2}$, then there exists a constant $C>0$ such that for all $r \in\left[1, \frac{1+\alpha}{2}\right)$,

$$
\|J(v)(t, \cdot)\|_{p} \leq C \int_{0}^{t}(t-s)^{-\frac{2-r}{\alpha}}\|v(s, \cdot)\|_{\rho} \mathrm{d} s, \quad \forall t \in[0, T] .
$$

Lemma 3.2. ([6,26]) If $H>\frac{1}{2}$, then

$$
L^{\frac{1}{H}}([0, T] \times \mathbb{R}) \subset \mathcal{H} .
$$

Next, we will mainly prove Theorem 3.1 by the fixed point principle. Let $\mathbb{B}$ be the space of all $L^{p}(\mathbb{R})$-valued $\mathcal{F}_{t}$-adapted processes $u(t, \cdot):[0, T] \times \mathbb{R} \longrightarrow \mathbb{R}$ with the norm

$$
\|u\|_{\mathbb{B}}:=\left[\sup _{0 \leq t \leq T} e^{-\eta t} \mathbb{E}\left[\|u(t, \cdot)\|_{p}^{p}\right]\right]^{\frac{1}{p}}, \quad \eta>0,
$$

with $\|\cdot\|_{p}$ the usual norm of $L^{p}(\mathbb{R})$. Then $\left(\mathbb{B},\|\cdot\|_{\mathbb{B}}\right)$ forms a Banach space. Further, for $u \in \mathbb{B}$, let us define an operator $\mathcal{A}_{\alpha}$ by (as follows)

$$
\mathcal{A}_{\alpha}(t, x):=\sum_{i=1}^{3} \mathcal{A}_{\alpha}^{i}(u)(t, x),
$$


where

$$
\begin{aligned}
& \mathcal{A}_{\alpha}^{1}(u)(t, x)=\int_{\mathbb{R}} G_{\alpha}(t, x-y) u_{0}(y) \mathrm{d} y, \\
& \mathcal{A}_{\alpha}^{2}(u)(t, x)=\int_{0}^{t} \int_{\mathbb{R}} G_{\alpha}(t-s, x-y) f(s, y, u(s, y)) \mathrm{d} y \mathrm{~d} s, \\
& \mathcal{A}_{\alpha}^{3}(u)(t, x)=\int_{0}^{t} \int_{\mathbb{R}} G_{\alpha}(t-s, x-y) \sigma(s, y) B^{H}(\mathrm{~d} y, \mathrm{~d} s) .
\end{aligned}
$$

Proposition 3.1. Under the assumptions of Theorem 3.1, for each $p>\frac{2(\alpha+1)}{\alpha-1}$ and $u \in \mathbb{B}$, it holds that $\mathcal{A}_{\alpha}(u) \in \mathbb{B}$.

Proof. From (6) of Lemma A.1, Corollary A.2 and the Young inequality, we obtain that

$$
\begin{aligned}
\left\|\mathcal{A}_{\alpha}^{1}(u)(t, x)\right\|_{p} & =\left\|\int_{\mathbb{R}} G_{\alpha}(t, x-y) u_{0}(y) \mathrm{d} y\right\|_{p} \\
& \leq t^{-\frac{1}{\alpha}}\left\|\int_{\mathbb{R}} G_{\alpha}\left(1, t^{-\frac{1}{\alpha}}(\cdot-y)\right) u_{0}(y) \mathrm{d} y\right\|_{p} \\
& \leq t^{-\frac{1}{\alpha}}\left\|\left[G_{\alpha}\left(1, t^{-\frac{1}{\alpha}} \cdot\right) * u_{0}(\cdot)\right](\cdot)\right\|_{p} \\
& \leq t^{-\frac{1}{\alpha}}\left\|G_{\alpha}\left(1, t^{-\frac{1}{\alpha}} \cdot\right)\right\|_{1} \cdot\left\|u_{0}(\cdot)\right\|_{p} \\
& \leq C\left\|u_{0}(\cdot)\right\|_{p}<\infty
\end{aligned}
$$

which is due to the fact that $\mathbb{E}\left[\left\|u_{0}(\cdot)\right\|_{p}^{p}\right]<\infty$.

Now let us consider $\mathcal{A}_{\alpha}^{2}(u)(t, x)$. Applying (1) of Lemma 3.1 with $\frac{1}{r}=\frac{1}{p}-\frac{1}{\rho}+1=1$ and condition (2) of Theorem 3.1, we conclude that

$$
\begin{aligned}
\mathbb{E}\left[\left\|\mathcal{A}_{\alpha}^{2}(u)(t, x)\right\|_{p}^{p}\right] & \leq C \mathbb{E}\left[\int_{0}^{t}(t-s)^{-\frac{1-r}{\alpha}}\|f(s, \cdot, u(s, \cdot))\|_{p} \mathrm{~d} s\right]^{p} \\
& \leq C \mathbb{E}\left[\int_{0}^{t}\left(1+\|u(s, \cdot)\|_{p}\right) \mathrm{d} s\right]^{p} \\
& \leq C_{p, T}\left[1+\sup _{0 \leq t \leq T} \mathbb{E}\|u(s, \cdot)\|_{p}^{p}\right] \\
& \leq C_{p, T}\left[1+\|u(s, \cdot)\|_{\mathbb{B}}^{p}\right]<\infty,
\end{aligned}
$$

since $u \in \mathbb{B}$.

In what follows, now we turn to $\mathcal{A}_{\alpha}^{3}(u)(t, x)$. Noticing that $L^{\frac{1}{H}}([0, T] \times \mathbb{R}) \subset \mathcal{H}$ from Lemma 3.2 when $H>\frac{1}{2}$, we deduce that

$$
\mathbb{E}\left\|\mathcal{A}_{\alpha}^{3}(u)(t, x)\right\|_{p}^{p}=\int_{\mathbb{R}} \mathbb{E}\left|\int_{0}^{t} \int_{\mathbb{R}} G_{\alpha}(t-s, x-y) \sigma(s, y) B^{H}(\mathrm{~d} y, \mathrm{~d} s)\right|^{p} \mathrm{~d} x
$$




$$
\begin{aligned}
& =\int_{\mathbb{R}} \mathbb{E}\left|\int_{0}^{t} \int_{\mathbb{R}}\left(\mathcal{K}_{H}^{*} G_{\alpha} \sigma(t-\cdot, x-\cdot)\right)(s, y) W(\mathrm{~d} y, \mathrm{~d} s)\right|^{p} \mathrm{~d} x \\
& \leq C_{p} \int_{\mathbb{R}}\left\langle\mathcal{K}_{H}^{*} G_{\alpha} \sigma(t-\cdot, x-\cdot), \mathcal{K}_{H}^{*} G_{\alpha} \sigma(t-\cdot, x-\cdot)\right\rangle_{L^{2}([0, T] \times \mathbb{R})}^{\frac{p}{2}} \mathrm{~d} x \\
& =C_{p} \int_{\mathbb{R}}\left\langle G_{\alpha} \sigma(t-\cdot, x-\cdot), G_{\alpha} \sigma(t-\cdot, x-\cdot)\right\rangle_{\mathcal{H}}^{\frac{p}{2}} \mathrm{~d} x \\
& \leq C_{p} \int_{\mathbb{R}}\left\|G_{\alpha} \sigma(t-\cdot, x-\cdot)\right\|_{L^{\frac{1}{H}}([0, T] \times \mathbb{R})}^{p} \mathrm{~d} x .
\end{aligned}
$$

By using the Hölder inequality, it follows that

$$
\begin{aligned}
& \left\|G_{\alpha} \sigma(t-\cdot, x-\cdot)\right\|_{L^{\frac{1}{H}([0, T] \times \mathbb{R})}}^{p} \mathrm{~d} x \\
& =\left[\int_{0}^{t} \int_{\mathbb{R}}\left|G_{\alpha}(t-s, x-y) \sigma(s, y)\right|^{\frac{1}{H}} \mathrm{~d} y \mathrm{~d} s\right]^{p H} \\
& \leq\left[\int_{0}^{t} \int_{\mathbb{R}}\left|G_{\alpha}(t-s, x-y)\right|^{\frac{1}{H}}|\sigma(s, y)|^{\frac{1}{H}} \mathrm{~d} y \mathrm{~d} s\right]^{p H} \\
& \leq\left[\int_{0}^{t}\left(\int_{\mathbb{R}}\left|G_{\alpha}(t-s, x-y)\right|^{\frac{1}{H} \cdot 2 H} \mathrm{~d} y\right)^{\frac{1}{2 H}} \cdot\left(\int_{\mathbb{R}}|\sigma(t-s, x-y)|^{\frac{2}{2 H-1}} \mathrm{~d} y\right)^{\frac{2 H-1}{2 H}} \mathrm{~d} s\right]^{p H} \\
& \leq\left[\int_{0}^{t}\left(\int_{\mathbb{R}}(t-s)^{-\frac{2}{\alpha}}\left|G_{\alpha}\left(1,(t-s)^{-\frac{2}{\alpha}}(x-y)\right)\right|^{2} \mathrm{~d} y\right)^{\frac{1}{2 H}} \cdot\left(\int_{\mathbb{R}}|\sigma(t-s, x-y)|^{\frac{2}{2 H-1}} \mathrm{~d} y\right)^{\frac{2 H-1}{2 H}} \mathrm{~d} s\right]^{p H} \\
& \leq\left[\int_{0}^{t}\left(\int_{\mathbb{R}}(t-s)^{-\frac{2}{\alpha}+\frac{1}{\alpha}}\left|G_{\alpha}(1, z)\right|^{2} \mathrm{~d} z\right)^{\frac{1}{2 H}} \cdot\left(\int_{\mathbb{R}}|\sigma(t-s, x-y)|^{\frac{2}{2 H-1}} \mathrm{~d} y\right)^{\frac{2 H-1}{2 H}} \mathrm{~d} s\right]^{p H} \\
& \leq\left[C_{\alpha, H} \int_{0}^{t}(t-s)^{-\frac{1}{2 \alpha H}}\left(\int_{\mathbb{R}} \frac{1}{\left(1+|z|^{\alpha+1}\right)^{2}} \mathrm{~d} z\right)^{\frac{1}{2 H}} \cdot\left(\int_{\mathbb{R}}|\sigma(t-s, x-y)|^{\frac{2}{2 H-1}} \mathrm{~d} y\right)^{\frac{2 H-1}{2 H}} \mathrm{~d} s\right]^{p H} \\
& \leq\left[C_{\alpha, H} \int_{0}^{t}(t-s)^{-\frac{1}{2 \alpha H}} \cdot\left(\int_{\mathbb{R}}|\sigma(t-s, x-y)|^{\frac{2}{2 H-1}} \mathrm{~d} y\right)^{\frac{2 H-1}{2 H}} \mathrm{~d} s\right]^{p H} \\
& \leq C_{\alpha, p, H}\left(\int_{0}^{t}(t-s)^{-\frac{1}{2 \alpha H} \cdot 2 H} \mathrm{~d} s\right)^{\frac{p}{2}} \cdot\left(\int_{0}^{t} \int_{\mathbb{R}}|\sigma(t-s, x-y)|^{\frac{2}{H H-1}} \mathrm{~d} y \mathrm{~d} s\right)^{\frac{2 H-1}{2} \cdot p} \\
& \leq C\left[\int_{0}^{t}(t-s)^{-\frac{1}{\alpha}} \mathrm{d} s\right]^{\frac{p}{2}} \cdot\|\sigma(\cdot, \cdot)\|_{L^{2}([0, T] \times \mathbb{R})}^{p}<\infty,
\end{aligned}
$$

where we have used the fact that $\sigma \in L^{\frac{2}{2 H-1}}([0, T] \times \mathbb{R}) \subset L^{2}([0, T] \times \mathbb{R})$ when $H>\frac{1}{2}$, and under the assumption $1-\frac{1}{\alpha}>0$. So we have $\mathcal{A}_{\alpha}^{3}(u)(t, x) \in \mathbb{B}$ for $p \geq 2$.

Therefore we have proved that the operator $\mathcal{A}_{\alpha}$ defined by (3.8) is an operator from $\mathbb{B}$ to itself. The proof is complete. 
In what follows, we will prove that the operator $\mathcal{A}_{\alpha}: \mathbb{B} \mapsto \mathbb{B}$ is a contraction operator. Proposition 3.2. ([6]) For $p>\frac{2(\alpha+1)}{\alpha-1}$, the operator $\mathcal{A}_{\alpha}$ is a contraction on $\mathbb{B}$ under the conditions of Theorem 3.1. That is to say, there exists a constant $\lambda \in(0,1)$ such that

$$
\left\|\mathcal{A}_{\alpha}(u)-\mathcal{A}_{\alpha}(v)\right\|_{\mathbb{B}} \leq \lambda\|u-v\|_{\mathbb{B}}, \quad \text { for } u, v \in \mathbb{B} .
$$

Proof. Assume that $u_{0}$ and $v_{0}$ are initials of $\left(\mathcal{F}_{t}\right)_{t \geq 0}$-adapted random fields $u, v \in \mathbb{B}$ such that $u_{0}=v_{0}$. Let us begin by considering $\mathcal{A}_{\alpha}^{1}(u)$. Note that, for $\rho=\frac{p}{3}$, by (1) of Lemma 3.1 with $\frac{1}{r}=\frac{1}{p}-\frac{1}{\rho}+1=1-\frac{2}{p}$ and condition (1) of Theorem 3.1, we have

$$
\begin{aligned}
& \mathbb{E}\left[\left\|\mathcal{A}_{\alpha}^{2}(u)(t, \cdot)-\mathcal{A}_{\alpha}^{2}(v)(t, \cdot)\right\|_{p}^{p}\right] \\
\leq & C \mathbb{E}\left[\int_{0}^{t}(t-s)^{-\frac{1}{\alpha}(1-r)}\|f(s, y, u(s, y))-f(s, y, v(s, y))\|_{p} \mathrm{~d} s\right]^{p} \\
\leq & C_{p}\left[\int_{0}^{t}(t-s)^{-\frac{1}{\alpha}(1-r)} \mathbb{E}\|u(s, \cdot)-v(s, \cdot)\|_{p} \mathrm{~d} s\right]^{p} .
\end{aligned}
$$

Thus

$$
\begin{aligned}
& \left\|\mathcal{A}_{\alpha}^{2}(u)(t, \cdot)-\mathcal{A}_{\alpha}^{2}(v)(t, \cdot)\right\|_{\mathbb{B}}^{p} \\
= & \sup _{0 \leq t \leq T} e^{-\eta t} \mathbb{E}\left[\left\|\mathcal{A}_{\alpha}^{2}(u)(t, \cdot)-\mathcal{A}_{\alpha}^{2}(v)(t, \cdot)\right\|_{p}^{p}\right] \\
\leq & C \sup _{0 \leq t \leq T} \mathbb{E}\left[\int_{0}^{t} e^{-\frac{\eta}{p}(t-s)}(t-s)^{-\frac{1}{\alpha}(1-r)} e^{-\frac{\eta}{p} s}\|u(s, \cdot)-v(s, \cdot)\|_{p} \mathrm{~d} s\right]^{p} \\
\leq & C_{p} \sup _{0 \leq t \leq T} \mathbb{E}\left[\int_{0}^{t} e^{-\eta s}\|u(s, \cdot)-v(s, \cdot)\|_{p}^{p} \mathrm{~d} s\right]\left[\int_{0}^{t}\left(e^{-\frac{\eta}{p}(t-s)}(t-s)^{-\frac{1}{\alpha}(1-r)}\right)^{\frac{p}{p-1}} \mathrm{~d} s\right]^{p-1} \\
\leq & C_{p} \sup _{0 \leq t \leq T}\left[\int_{0}^{t} e^{-\eta s} \mathbb{E}\|u(s, \cdot)-v(s, \cdot)\|_{p}^{p} \mathrm{~d} s\right]\left[\int_{0}^{t}\left(e^{-\frac{\eta}{p}(t-s)}(t-s)^{-\frac{1}{\alpha}(1-r)}\right)^{\frac{p}{p-1}} \mathrm{~d} s\right]^{p-1} \\
= & C_{p} T \omega(p, t)\|u-v\|_{\mathbb{B}}^{p},
\end{aligned}
$$

where

$$
\omega(p, t)=\left[\int_{0}^{t}\left(e^{-\frac{\eta}{p}(t-s)}(t-s)^{-\frac{1}{\alpha}(1-r)}\right)^{\frac{p}{p-1}} \mathrm{~d} s\right]^{p-1} .
$$

Let $\tau=\frac{p}{p-1} \frac{1}{\alpha}(1-r)$, then

$$
\begin{aligned}
\omega(p, t) & \leq\left[\int_{0}^{+\infty}\left(e^{-\frac{\eta}{p}(t-s)}(t-s)^{-\frac{1}{\alpha}(1-r)}\right)^{\frac{p}{p-1}} \mathrm{~d} s\right]^{p-1} \\
& =\left[\frac{(p-1)^{\tau+1}}{\eta^{\tau+1}} \int_{0}^{+\infty} e^{-x} x^{\tau} \mathrm{d} x\right]^{p-1}
\end{aligned}
$$




$$
=\left[\frac{(p-1)^{\tau+1}}{\eta^{\tau+1}} \Gamma(\tau+1)\right]^{p-1},
$$

with $p \geq 3$. So

$$
\begin{aligned}
& \left\|\mathcal{A}_{\alpha}^{2}(u)(t, \cdot)-\mathcal{A}_{\alpha}^{2}(v)(t, \cdot)\right\|_{\mathbb{B}} \\
\leq & C_{p} T^{\frac{1}{p}}\left[\frac{(p-1)^{\tau+1}}{\eta^{\tau+1}} \Gamma(\tau+1)\right]^{\frac{p-1}{p}} \cdot\|u-v\|_{\mathbb{B}} \leq \lambda\|u-v\|_{\mathbb{B}},
\end{aligned}
$$

with $\lambda \in(0,1)$ by choosing $\eta>0$ large enough, then $\mathcal{A}_{\alpha}^{2}$ is a contraction on $\mathbb{B}$. Meanwhile, we can quickly conclude from the expression for $\mathcal{A}_{\alpha}^{3}$ that $\mathcal{A}_{\alpha}^{3}$ is also a contraction on $\mathbb{B}$.

Therefore, it follows from (3.8) that $\mathcal{A}_{\alpha}(\cdot)$ is a contraction on $\mathbb{B}$ if $\eta>0$ large enough. Thus the proof of Proposition 3.2 is complete.

Motivated by Propositions 3.1 and 3.2 and the fixed point principle on the set $\{u \in \mathbb{B}$ : $\left.u(0)=u_{0}\right\}$, we conclude that Eq. (1.1) admits a unique solution $u \in \mathbb{B}$. Thus the conclusion of Theorem 3.1 follows.

\section{The averaging principle}

In this section, we are going to prove the averaging principle of solutions for SPDEs with fractional noises. Consider the Eq. (1.2) in the following form:

$$
\begin{gathered}
u_{\varepsilon}(t, x)=\int_{\mathbb{R}} G_{\alpha}(t, x-y) u_{0}(y) \mathrm{d} y+\int_{0}^{t} \int_{\mathbb{R}} G_{\alpha}(t-s, x-y) \sigma\left(\frac{s}{\varepsilon}, y\right) B^{H}(\mathrm{~d} s, \mathrm{~d} y) \\
+\int_{0}^{t} \int_{\mathbb{R}} G_{\alpha}(t-s, x-y) f\left(s, y, u_{\varepsilon}(s, y)\right) \mathrm{d} y \mathrm{~d} s .
\end{gathered}
$$

According to Theorem 3.1, Eq. (4.1) also has a unique mild solution $u_{\varepsilon}(t, x), t \in[0, T]$ for $\varepsilon>0$. We will examine whether the solution process $u_{\varepsilon}(t, x)$ can be approximated to the solution process $\bar{u}(t, x)$ of the simplified equation:

$$
\begin{gathered}
\bar{u}(t, x)=\int_{\mathbb{R}} G_{\alpha}(t, x-y) u_{0}(y) \mathrm{d} y+\int_{0}^{t} \int_{\mathbb{R}} G_{\alpha}(t-s, x-y) \bar{\sigma}(y) B^{H}(\mathrm{~d} s, \mathrm{~d} y) \\
+\int_{0}^{t} \int_{\mathbb{R}} G_{\alpha}(t-s, x-y) f(s, y, \bar{u}(s, y)) \mathrm{d} y \mathrm{~d} s .
\end{gathered}
$$

Based on the assumptions of Theorem 3.1, further, we make the following assumptions throughout this section:

Assumption 4.1. $\sigma(s, y): \mathbb{R}_{+} \times \mathbb{R} \rightarrow \mathbb{R}$ is measurable, bounded, and

$$
\left|\sigma\left(s, y_{1}\right)-\sigma\left(s, y_{2}\right)\right| \leq L_{\sigma}\left|y_{1}-y_{2}\right|,
$$


where $L_{\sigma}$ is a constant. Assume that there exist the following limit

$$
\bar{\sigma}(y)=\lim _{t \rightarrow \infty} \frac{1}{t} \int_{0}^{t} \sigma(s, y) \mathrm{d} s .
$$

It is easy to see that $\bar{\sigma}(y)$ satisfies the Assumption 4.1.

Assumption 4.2. Function $G_{\sigma}(z, y)=\int_{0}^{z}(\sigma(s, y)-\bar{\sigma}(y))^{\frac{1}{H}} \mathrm{~d} s, z \in \mathbb{R}_{+}, y \in \mathbb{R}$ is bounded.

Lemma 4.1. Let $h(z, y)$ and $\bar{h}(y)$ be measurable and functions

$$
K(z, y)=(h(z, y)-\bar{h}(y))^{\frac{1}{H}} \text { and } G(z, y)=\int_{0}^{z}(h(v, y)-\bar{h}(y))^{\frac{1}{H}} \mathrm{~d} v, \quad z \in \mathbb{R}_{+}, \quad y \in \mathbb{R}
$$

are bounded. Then

$$
\left.\left.\sup _{y \in \mathbb{R}, s>0, t \in[0, T]}\left|\frac{1}{\varepsilon^{\gamma}} \int_{0}^{t} \int_{\mathbb{R}}\right| G_{\alpha}(t-s, x-y)(h(s / \varepsilon, y)-\bar{h}(y))\right|^{\frac{1}{H}} \mathrm{~d} s\right|^{p H}<+\infty,
$$

where $\gamma$ is a constant relevant to $p, H$.

Proof. Using the substitution $v=(t-s) / \varepsilon$, we obtain

$$
\begin{aligned}
& \left(\int_{0}^{t} \int_{\mathbb{R}}\left|G_{\alpha}(t-s, x-y)\left(h\left(\frac{s}{\varepsilon}, y\right)-\bar{h}(y)\right)\right|^{\frac{1}{H}} \mathrm{~d} y \mathrm{~d} s\right)^{p H} \\
\leq & \left(\int_{0}^{t} \int_{\mathbb{R}}\left|G_{\alpha}(t-s, x-y)\right|^{\frac{1}{H}}\left|\left(h\left(\frac{s}{\varepsilon}, y\right)-\bar{h}(y)\right)\right|^{\frac{1}{H}} \mathrm{~d} y \mathrm{~d} s\right)^{p H} \\
= & \left(\varepsilon \int_{0}^{\frac{t}{\varepsilon}} \int_{\mathbb{R}}\left|G_{\alpha}(v \varepsilon, x-y)\right|^{\frac{1}{H}}\left|\left(h\left(\frac{t}{\varepsilon}-v, y\right)-\bar{h}(y)\right)\right|^{\frac{1}{H}} \mathrm{~d} y \mathrm{~d} v\right)^{p H} \\
= & \varepsilon^{p H}\left(\int_{0}^{\frac{t}{\varepsilon}} \sup _{y}\left|\left(h\left(\frac{t}{\varepsilon}-v, y\right)-\bar{h}(y)\right)\right|^{\frac{1}{H}}\left(\int_{\mathbb{R}}\left|G_{\alpha}(v \varepsilon, x-y)\right|^{\frac{1}{H}} \mathrm{~d} y\right) \mathrm{d} v\right)^{p H} .
\end{aligned}
$$

Under the assumption $1-\frac{1}{\alpha H}+\frac{1}{\alpha}>0$, we have

$$
\begin{aligned}
& \int_{\mathbb{R}}\left|G_{\alpha}(t-s, x-y)\right|^{\frac{1}{H}} \mathrm{~d} y \leq(t-s)^{-\frac{1}{\alpha H}} \int_{\mathbb{R}}\left|G_{\alpha}\left(1,(t-s)^{-\frac{1}{\alpha H}}(x-y)\right)\right|^{\frac{1}{H}} \mathrm{~d} y \\
\leq & (t-s)^{-\frac{1}{\alpha H}+\frac{1}{\alpha}} \int_{\mathbb{R}}\left|G_{\alpha}(1, z)\right|^{\frac{1}{H}} \mathrm{~d} y \leq(t-s)^{-\frac{1}{\alpha H}+\frac{1}{\alpha}} \int_{\mathbb{R}} \frac{1}{\left(1+|z|^{1+\alpha}\right)^{\frac{1}{H}}} \mathrm{~d} y \\
\leq & C_{\alpha, H}(t-s)^{-\frac{1}{\alpha}+\frac{1}{\alpha H}} .
\end{aligned}
$$

Then, we can obtain

$$
\left(\int_{0}^{t} \int_{\mathbb{R}}\left|G_{\alpha}(t-s, x-y)\left(h\left(\frac{s}{\varepsilon}, y\right)-\bar{h}(y)\right)\right|^{\frac{1}{H}} \mathrm{~d} y \mathrm{~d} s\right)^{p H}
$$




$$
\leq C_{\alpha, H} \varepsilon^{p H}\left(\int_{0}^{\frac{t}{\varepsilon}} \sup _{y}\left|\left(h\left(\frac{t}{\varepsilon}-v, y\right)-\bar{h}(y)\right)\right|^{\frac{1}{H}} \cdot(v \varepsilon)^{-\frac{1}{\alpha H}+\frac{1}{\alpha}} \mathrm{d} v\right)^{p H} .
$$

Let $|h(z, y)-\bar{h}(y)| \leq C_{h}$, and we can denote

$$
F_{\varepsilon}(z)=\int_{0}^{z}|h(t / \varepsilon-v, y)-\bar{h}(y)|^{\frac{1}{H}} \mathrm{~d} v, \quad 0 \leq r \leq t / \varepsilon .
$$

Then $F_{\varepsilon}(z)$ is bounded. Denoting $\left|F_{\varepsilon}(z)\right| \leq C_{F}$, where $C_{F}$ does not depend on $\varepsilon$, we have

$$
\begin{aligned}
& \int_{0}^{\frac{t}{\varepsilon}} \sup _{y}\left|\left(h\left(\frac{t}{\varepsilon}-v, y\right)-\bar{h}(y)\right)\right|^{\frac{1}{H}} \cdot(v \varepsilon)^{-\frac{1}{\alpha H}+\frac{1}{\alpha}} \mathrm{d} v \\
\leq & \sup _{y} \int_{0}^{\frac{t}{\varepsilon}}\left|\left(h\left(\frac{t}{\varepsilon}-v, y\right)-\bar{h}(y)\right)\right|^{\frac{1}{H}} \cdot(v \varepsilon)^{-\frac{1}{\alpha H}+\frac{1}{\alpha}} \mathrm{d} v \\
\leq & \sup _{y} \int_{0}^{1}\left|\left(h\left(\frac{t}{\varepsilon}-v, y\right)-\bar{h}(y)\right)\right|^{\frac{1}{H}} \cdot(v \varepsilon)^{-\frac{1}{\alpha H}+\frac{1}{\alpha}} \mathrm{d} v \\
\quad & \quad \sup _{y} \int_{1}^{\frac{t}{\varepsilon}}\left|\left(h\left(\frac{t}{\varepsilon}-v, y\right)-\bar{h}(y)\right)\right|^{\frac{1}{H}} \cdot(v \varepsilon)^{-\frac{1}{\alpha H}+\frac{1}{\alpha}} \mathrm{d} v \\
= & : B_{1}+B_{2} .
\end{aligned}
$$

Noticing that

$$
\begin{aligned}
B_{1} & =\sup _{y} \int_{0}^{1}\left|\left(h\left(\frac{t}{\varepsilon}-v, y\right)-\bar{h}(y)\right)\right|^{\frac{1}{H}} \cdot(v \varepsilon)^{-\frac{1}{\alpha H}+\frac{1}{\alpha}} \mathrm{d} v \\
& \leq C_{h, H} \int_{0}^{1}(v \varepsilon)^{-\frac{1}{\alpha H}+\frac{1}{\alpha}} \mathrm{d} v=C_{h, \alpha, H^{\varepsilon}} \varepsilon^{-\frac{1}{\alpha H}+\frac{1}{\alpha}}
\end{aligned}
$$

For $B_{2}$, using the integration by parts and inequality $|a-b| \leq|a|+|b|$, we obtain

$$
\begin{aligned}
B_{2} & =\sup _{y} \int_{1}^{\frac{t}{\varepsilon}}\left|\left(h\left(\frac{t}{\varepsilon}-v, y\right)-\bar{h}(y)\right)\right|^{\frac{1}{H}} \cdot(v \varepsilon)^{-\frac{1}{\alpha H}+\frac{1}{\alpha}} \mathrm{d} v \\
& =\int_{1}^{\frac{t}{\varepsilon}}\left|(v \varepsilon)^{-\frac{1}{\alpha}+\frac{1}{\alpha H}} \mathrm{~d} F_{\varepsilon}(v)\right| \\
& =\left|(v \varepsilon)^{-\frac{1}{\alpha H}+\frac{1}{\alpha}} \cdot F_{\varepsilon}(v)\right|_{1}^{\frac{t}{\varepsilon}}-\int_{1}^{\frac{t}{\varepsilon}} F_{\varepsilon}(v) \cdot \varepsilon\left(-\frac{1}{\alpha H}+\frac{1}{\alpha}-1\right)\left((v \varepsilon)^{-\frac{1}{\alpha H}+\frac{1}{\alpha}}\right) \mathrm{d} v \mid \\
& \leq C_{F}\left|t^{-\frac{1}{\alpha H}+\frac{1}{\alpha}}-\varepsilon^{-\frac{1}{\alpha}+\frac{1}{\alpha H}}\right|+C_{F}\left|t^{-\frac{1}{\alpha H}+\frac{1}{\alpha}}-\varepsilon^{-\frac{1}{\alpha H}+\frac{1}{\alpha}}\right| \\
& =2 C_{F} t^{-\frac{1}{\alpha H}+\frac{1}{\alpha}}-2 C_{F} \varepsilon^{-\frac{1}{\alpha H}+\frac{1}{\alpha}}
\end{aligned}
$$




$$
\leq C_{F, T}-2 C_{F} \varepsilon^{-\frac{1}{\alpha H}+\frac{1}{\alpha}} .
$$

And then, by (4.3), (4.4) and the inequality $(a+b)^{p} \leq 2^{p-1}\left(a^{p}+b^{p}\right)$, we get

$$
\begin{aligned}
& \left(\int_{0}^{t} \int_{\mathbb{R}}\left|G_{\alpha}(t-s, x-y)\left(h\left(\frac{s}{\varepsilon}, y\right)-\bar{h}(y)\right)\right|^{\frac{1}{H}} \mathrm{~d} y \mathrm{~d} s\right)^{p H} \\
\leq & \varepsilon^{p H}\left(C_{h, H} \mathcal{\varepsilon}^{-\frac{1}{\alpha H}+\frac{1}{\alpha}}+C_{F, T}-2 C_{F} \mathcal{E}^{-\frac{1}{\alpha H}+\frac{1}{\alpha}}\right)^{p H} \\
\leq & \varepsilon^{p H}\left(C_{1} \varepsilon^{-\frac{1}{\alpha H}+\frac{1}{\alpha}}+C_{F, T}\right)^{p H} \\
\leq & 2^{p H-1} \mathcal{E}^{p H}\left(C_{2} \varepsilon^{\left(-\frac{1}{\alpha H}+\frac{1}{\alpha}\right) p H}+C_{3}\right) \\
\leq & C_{4} \varepsilon^{\left(-\frac{1}{\alpha H}+\frac{1}{\alpha}\right) p H+p H}+C_{5} \varepsilon^{p H} \\
\leq & C \varepsilon^{\left(-\frac{1}{\alpha H}+\frac{1}{\alpha}+1\right) p H},
\end{aligned}
$$

where $C_{1}=C_{h, H}-2 C_{F},\left(C_{F, T}\right)^{p H}=C_{3}, 2^{p H-1} C_{2}=C_{4}, 2^{p H-1} C_{3}=C_{5}, C=\max \left\{C_{4}, C_{5}\right\}$. So when $\gamma=\left(-\frac{1}{\alpha H}+\frac{1}{\alpha}+1\right) p H$, we can get

$$
\left.\left.\sup _{y \in \mathbb{R}, s>0, t \in[0, T]}\left|\frac{1}{\varepsilon^{\gamma}} \int_{0}^{t} \int_{\mathbb{R}}\right| G_{\alpha}(t-s, x-y)(h(s / \varepsilon, y)-\bar{h}(y))\right|^{\frac{1}{H}} \mathrm{~d} s\right|^{p H}<+\infty .
$$

Thus, we complete the proof of this Lemma.

Remark 4.1. From Assumptions 4.1-4.2 and Lemma 4.1, it follows from that

$$
\left(\int_{0}^{t} \int_{\mathbb{R}}\left|G_{\alpha}(t-s, x-y)\left(\sigma\left(\frac{s}{\varepsilon}, y\right)-\bar{\sigma}(y)\right)\right|^{\frac{1}{H}} \mathrm{~d} y \mathrm{~d} s\right)^{p H} \leq C \varepsilon^{\gamma} .
$$

Theorem 4.1. Assume that the conditions of Theorem 3.1 and Assumptions 4.1-4.2 hold. Then there exist versions of $u_{\varepsilon}$ and $\bar{u}$ such that for $\gamma=p H$,

$$
\sup _{\varepsilon>0, t \in[0, T], x \in \mathbb{R}} \varepsilon^{-\gamma} \mathbb{E}\left|u_{\varepsilon}(t, x)-\bar{u}(t, x)\right|^{p}<+\infty \text {, a.s. }
$$

Proof. Using the inequality $(a+b)^{p} \leq 2^{p-1}\left(a^{p}+b^{p}\right),(4.1)$ and (4.2), we have

$$
\begin{aligned}
& \mathbb{E}\left|u_{\varepsilon}(t, x)-\bar{u}(t, x)\right| \\
= & \mathbb{E} \mid \int_{0}^{t} \int_{\mathbb{R}} G_{\alpha}(t-s, x-y)\left(\sigma\left(\frac{s}{\varepsilon}, y\right)-\bar{\sigma}(y)\right) B^{H}(\mathrm{~d} s, \mathrm{~d} y) \\
\quad & \quad \int_{0}^{t} \int_{\mathbb{R}} G_{\alpha}(t-s, x-y) f\left(s, y, u_{\varepsilon}(s, y)\right)-\left.f(s, y, \bar{u}(s, y)) \mathrm{d} y \mathrm{~d} s\right|^{p} \\
\leq & 2^{p-1} \mathbb{E}\left|\int_{0}^{t} \int_{\mathbb{R}} G_{\alpha}(t-s, x-y)\left(\sigma\left(\frac{s}{\varepsilon}, y\right)-\bar{\sigma}(y)\right) B^{H}(\mathrm{~d} s, \mathrm{~d} y)\right|^{p}
\end{aligned}
$$




$$
\begin{aligned}
& \quad+2^{p-1} \mathbb{E}\left|\int_{0}^{t} \int_{\mathbb{R}} G_{\alpha}(t-s, x-y) f\left(s, y, u_{\varepsilon}(s, y)\right)-f(s, y, \bar{u}(s, y)) \mathrm{d} y \mathrm{~d} s\right|^{p} \\
& =: I_{1}+I_{2} .
\end{aligned}
$$

For $I_{1}$, by inequality (3.11) and Remark 4.2, we deduce that

$$
\begin{aligned}
& I_{1}=\mathbb{E}\left|\int_{0}^{t} \int_{\mathbb{R}} G_{\alpha}(t-s, x-y)\left(\sigma\left(\frac{s}{\varepsilon}, y\right)-\bar{\sigma}(y)\right) B^{H}(\mathrm{~d} s, \mathrm{~d} y)\right|^{p} \\
& =\mathbb{E}\left|\int_{0}^{t} \int_{\mathbb{R}} \mathcal{K}^{*} G_{\alpha}(t-\cdot, x-\cdot)\left(\sigma\left(\frac{\dot{\varepsilon}}{\varepsilon}, \cdot\right)-\bar{\sigma}(\cdot)\right)(s, y) W(\mathrm{~d} s, \mathrm{~d} y)\right|^{p} \\
& \leq C_{p}\left\langle\mathcal { K } _ { H } ^ { * } G _ { \alpha } ( t - \cdot , x - \cdot ) \left(\sigma\left(\frac{\dot{\varepsilon}}{\varepsilon}, \cdot\right), \mathcal{K}_{H}^{*} G_{\alpha}(t-\cdot, x-\cdot)\left(\sigma\left(\frac{\dot{\varepsilon}}{\varepsilon}, \cdot\right)\right\rangle_{L^{2}([0, T] \times \mathbb{R})}^{\frac{p}{2}}\right.\right. \\
& =C_{p}\left\langle G_{\alpha}\left(t-\cdot, x-\cdot\left(\sigma\left(\frac{\dot{\varepsilon}}{\varepsilon}, \cdot\right)-\bar{\sigma}(\cdot)\right)\right), G_{\alpha}(t-\cdot, x-\cdot)\left(\sigma\left(\frac{\dot{\varepsilon}}{\varepsilon} \cdot\right)-\bar{\sigma}(\cdot)\right)\right\rangle_{\mathcal{H}}^{\frac{p}{2}} \\
& \leq C_{p}\left\|G_{\alpha}(t-\cdot, x-\cdot)\left(\sigma\left(\frac{\dot{\varepsilon}}{\varepsilon} \cdot\right)-\bar{\sigma}(\cdot)\right)\right\|_{L \frac{1}{H}([0, T] \times \mathbb{R})}^{p} \\
& =C_{p}\left(\int_{0}^{t} \int_{\mathbb{R}}\left|G_{\alpha}(t-s, x-y)\left(\sigma\left(\frac{s}{\mathcal{\varepsilon}}, y\right)-\bar{\sigma}(y)\right)\right|^{\frac{1}{H}} \mathrm{~d} y \mathrm{~d} s\right)^{p H} \\
& \leq C \varepsilon^{\gamma} \text {. }
\end{aligned}
$$

Then for $I_{2}$, owing to the equality $\int_{\mathbb{R}} G_{\alpha}(t-s, x-y) \mathrm{d} y=1$ and Hölder inequality, we have

$$
\begin{aligned}
I_{2} & =\mathbb{E}\left|\int_{0}^{t} \int_{\mathbb{R}} G_{\alpha}(t-s, x-y) f\left(s, y, u_{\varepsilon}(s, y)\right)-f(s, y, \bar{u}(s, y)) \mathrm{d} y \mathrm{~d} s\right|^{p} \\
& \leq L^{p} \mathbb{E}\left|\int_{0}^{t} \mathrm{~d} s \int_{\mathbb{R}} G_{\alpha}(t-s, x-y)\right| u_{\varepsilon}(s, y)-\bar{u}(s, y)|\mathrm{d} y|^{p} \\
& \leq L^{p} \mathbb{E}\left|\int_{0}^{t} \sup _{y}\right| u_{\varepsilon}(s, y)-\bar{u}(s, y)\left|\mathrm{d} s \int_{\mathbb{R}} G_{\alpha}(t-s, x-y) \mathrm{d} y\right|^{p} \\
& \leq L^{p} T^{p-1} \int_{0}^{t} \sup _{y} \mathbb{E}\left|u_{\varepsilon}(s, y)-\bar{u}(s, y)\right|^{p} \mathrm{~d} s \\
& \leq C_{L, T, p} \int_{0}^{t} \sup _{y} \mathbb{E}\left|u_{\varepsilon}(s, y)-\bar{u}(s, y)\right|^{p} \mathrm{~d} s .
\end{aligned}
$$

Finally, we get

$$
\sup _{\varepsilon>0, t \in[0, T], x \in \mathbb{R}} \mathbb{E}\left|u_{\varepsilon}(t, x)-\bar{u}(t, x)\right|^{p} \leq C \varepsilon^{\gamma}+C_{L, T, p} \int_{0}^{t} \sup _{y} \mathbb{E}\left|u_{\varepsilon}(s, y)-\bar{u}(s, y)\right|^{p} \mathrm{~d} s,
$$

by Gronwall's inequality, the above inequality can be expressed as

$$
\sup _{\varepsilon>0, t \in[0, T], x \in \mathbb{R}} \mathbb{E}\left|u_{\varepsilon}(t, x)-\bar{u}(t, x)\right|^{p} \leq C \varepsilon^{\gamma} .
$$

That finishes the proof. 


\section{A Appendix. The Green function $([6,23,24])$}

The fractional differential operator $D_{\alpha}^{\delta}$ is an extension of the inverse of the generalized Riesz-Feller potential when $\alpha>2$. It is given for $\alpha>0$ by the following Definition

Definition A.1. The fractional differential $D_{\alpha}^{\delta} \varphi$ is given by

$$
\begin{aligned}
& D_{\delta}^{\alpha} \varphi=F^{-1}\left\{\psi_{\alpha}(\lambda) F(\varphi(x), \lambda)\right\}, \\
& \psi_{\alpha}(\lambda)=-|\lambda|^{\alpha} e^{-i \delta \frac{\pi}{2} \operatorname{sgn}(\lambda)},
\end{aligned}
$$

$\delta \leq \min \left\{\alpha-[\alpha]_{2}, 2+[\alpha]_{2}-\alpha\right\},[\alpha]_{2}$ is the largest even integer less than or equal to $\alpha$ (even part of $\alpha$ ), and for $\alpha=0$ when $\alpha \in 2 N+1$, and $F$ (respectively $F^{-1}$ ) is the Fourier (respectively Fourier inverse) transform.

The operator $D_{\alpha}^{\delta}$ is a closed, densely defined operator on $L^{2}(\mathbb{R})$ and it is the infinitesimal generator of a semigroup which is not symmetric and not a contraction. This operator is a generalization of various well-known operators, such as the Laplacian operator (when $\alpha=2$ ), the inverse of the generalized Riesz-Feller potential (when $\alpha>2$ ), the Riemann-Liouville differential operator (when $\delta=2+[\alpha]_{2}-\alpha$ or $\delta=\alpha-[\alpha]_{2}$, see $[6,22,23,25]$ for more details). It is self-adjoint only when $\delta=0$ and in this case, it coincides with the fractional power of the Laplacian. We refer the readers to Debbi [22] and Debbi and Dozzi [23] for more details about this operator. Let the Green function $G_{\alpha}(t, x)$ associated with Eq. (1.1) be the fundamental solution of the Cauchy problem

$$
\left\{\begin{array}{l}
\frac{\partial}{\partial t} G_{\alpha}(t, x)=D_{\delta}^{\alpha} G_{\alpha}(t, x), \quad t>0, x \in \mathbb{R}, \\
G_{\alpha}(0, x)=\delta_{0}(x),
\end{array}\right.
$$

where $\delta_{0}$ is the Dirac distribution at the point zero. Using Fourier's calculus we obtain

$$
G_{\alpha}(t, x)=F^{-1}\left\{e^{\psi_{\alpha}(\lambda) t}\right\}=\frac{1}{2 \pi} \int_{\mathbb{R}} \exp \left(-i \lambda x-t|\lambda|^{\alpha} e^{-i \delta \frac{\pi}{2} \operatorname{sgn}(\lambda)}\right) \mathrm{d} \lambda .
$$

The function $G_{\alpha}(t, \cdot)$ has the following properties (see e.g., [22,23]).

Lemma A.1. For $\alpha \in(0, \infty) \backslash \mathbb{N}$

(1) $\int_{\mathbb{R}} G_{\alpha}(t, x) \mathrm{d} x=1$.

(2) $G_{\alpha}(t, x)$ is real but in general it is not symmetric relatively to $x$ and it is not everywhere positive.

(3) $G_{\alpha}(t, x)$ satisfies the semigroup property, or the Chapman-Kolmogorov equation, i.e. for $0<s<t$

$$
G_{\alpha}(t+s, x)=\int_{\mathbb{R}} G_{\alpha}(t, \xi) G_{\alpha}(s, x-\xi) \mathrm{d} \xi .
$$


(4) For $0<\alpha \leq 2$, the function $G_{\alpha}(t, \cdot)$ is the density of a Lévy stable process in time $t$.

(5) For fixed $t, G_{\alpha}(t, \cdot) \in C^{\infty}$ and $\frac{\partial^{\beta}}{\partial x^{\beta}} G_{\alpha}(t, x)$ is bounded and tends to zero when $|x|$ tends to $\infty$ for $\beta \in \mathbb{R}_{+}$.

(6) $\frac{\partial^{n}}{\partial x^{n}} G_{\alpha}(t, x)=\left.t^{-\frac{n+1}{\alpha}} \frac{\partial^{n}}{\partial \zeta^{n}} G_{\alpha}(1, \zeta)\right|_{\zeta=t^{-\frac{1}{\alpha}} x^{\prime}}$ for all $n \geq 0$ (when $n=0$, it is called the scaling property).

Remark A.1. The proof of this Lemma can be found in [23].

Corollary A.1. Let $\alpha \in(1,+\infty)$, then there exists a constant $K_{\alpha}$ such that

$$
\begin{aligned}
& \left|G_{\alpha}(1, x)\right| \leq K_{\alpha}\left(1+|x|^{1+\alpha}\right)^{-1}, \\
& \left|G_{\alpha}^{(n)}(1, x)\right| \leq K_{\alpha} \frac{1+|x|^{\alpha+n-1}}{\left(1+|x|^{\alpha+n}\right)^{2}} .
\end{aligned}
$$

Corollary A.2. Let $\alpha \in(1,+\infty)$, for $n \geq 1$, and $T \geq 0$, for $\gamma$ such that $\frac{1}{\alpha+n+1}<\gamma<\frac{\alpha+1}{n+1}$,

$$
\int_{0}^{T} \int_{\mathbb{R}}\left|\frac{\partial^{n}}{\partial x^{n}} G_{\alpha}(t, x)\right|^{\gamma} \mathrm{d} x \mathrm{~d} t<\infty .
$$

\section{Acknowledgement}

The authors thank the editors and the reviewers for their useful comments.

\section{References}

[1] Samko S. G., Kilbas A. A., Marichev O. I., Fractional Integrals and Derivatives. Theory and Applications, Gordon and Breach, London, 1993.

[2] Mueller C., The heat equation with Lévy noise. Stochastic Process. Appl. 74 (1998), 67-82.

[3] $\mathrm{Wu}$ J. L., Fractal Burgers equation with stable Lévy noise. International Conference SPDE and Applications-VII. (2004), 4-10.

[4] Bonaccorsi S., Tubaro L., Mittag-Leffler's function and stochastic linear Volterra equations of convolution type. Stoch. Anal. Appl. 21 (2003), 61-78.

[5] Cui J., Yan L., Existence result for fractional neutral stochastic integro-differential equations with infinite delay. J. Phys. A. 44 (2011), 335201.

[6] Liu J. F., Yan L. T., Cang Y. Q., On a jump-type stochastic fractional partial differential equation with fractional noises. Nonlinear Analysis. 75 (2012), 6060-6070.

[7] Khasminskii R. Z., A limit theorem for the solution of differential equations with random right-hand sides. Theory Probab. Appl. 11 (1963), 390-405.

[8] Khasminskii R. Z., On the principle of averaging the Itô stochastic differential equations. Kybernetika (Prague). 4 (1968), 260-279. 
[9] Xu Y., Duan J., Xu W., An averaging principle for stochastic dynamical systems with Lévy noise. Physica D: Nonlinear Phenomena. 240 (2011), 1395-1401.

[10] Xu J., Liu J., An averaging principle for multivalued stochastic differential equations. Stochastic Anal. Appl. 32 (2014), 962-974.

[11] Pei B., Xu Y., Wu J. L., Two-time-scales hyperbolic-parabolic equations driven by Poisson random measures: Existence, uniqueness and averaging principles. J. Math. Anal. Appl. 447 (2017), 243-268.

[12] Pei B., Xu Y., Yin G., Stochastic averaging for a class of two-time-scale systems of stochastic partial differential equations. Nonlinear Anal. 160 (2017), 159-176.

[13] Bao J., Yin G., Yuan C., Two-time-scale stochastic partial differential equations driven by $\alpha$-stable nioses: Averaging principles. Bernoulli. 23 (2017), 645-669.

[14] Radchenko V. M., Averaging principle for the heat equation driven by general stochastic measure. Stastics and Probability Letters. 146 (2019), 224-230.

[15] Duncan T. E., Maslowski B., Pasik-Duncan B., Fractional Brownian motion and stochastic equations in Hilbert spaces. Stoch. Dyn. 2 (2002), 225-250.

[16] Tindel S., Tudor C. A., Viens F., Stochastic evolution equations with fractional Brownian motion. Probab. Theory Related Fields. 127 (2003), 186-204.

[17] Boufoussi B., Hajji S., Neutral stochastic functional differential equations driven by a fractional Brownian motion in a Hilbert space. Statist, Probab. Lett. 82 (2012), 1549-1558.

[18] Caraballo T., Garrido-Atienza M. J., Taniguchi T., The existence and exponential behavior of solutions to stochastic delay evolution equations with a fractional Brownian motion. Nonlinear Analysis. 74 (2011), 3671-3684.

[19] Ren Y., Chen X., Sakthivel R., Impulsive neutral stochastic functional integro-differential equations with infinite delay driven by fBm. Applied Mathematics and Computation. 247 (2011), 205-212.

[20] Debbi L., On some properties of a high order fractional differential operator which is not in general selfadjoint. Appl. Math. Sci. 1 (2007), 1325-1339.

[21] Debbi L., Dozzi M., On the solutions of nonlinear stochastic fractional partial differential equations in one spatial dimension. Stoch. Proc. Appl. 115 (2005), 1764-1781.

[22] Walsh J. B., An introduction to stochastic partial differential equations. In: Ecole d'été de Probabilités de St. Flour XIV, in: Lect. Notes in Math, vol. 1180, Springer-Verlag, Berlin, 1986, 266-439.

[23] Albeverio J., Wu J., Zhang T., Parabolic SPDEs driven by Poisson white noise. Stochastic Process. Appl. 74 (1998), 21-36.

[24] Truman A., Wu J., Stochastic Burgers equation with Lévy space-time white noises. Probabilistic Methods in Fluids. (2003), 298-323.

[25] Ye H. P., Gao J. M., Ding Y. S., A generalized Gronwall inequality and its application to a fractional differential equation. J. Math. Anal. Appl. 328 (2007), 1075-1081.

[26] Mmin J., Mishura Y., Valkeila E., Inequalities for moments of Wiener integrals with respect to a fractional Brownian motion. Statist. Probab. Lett. 51 (2001), 197-206. 